REVIEW

\title{
Introduction to history taking and principles of sexual health
}

\author{
R Jones, S Barton
}

Postgrad Med J 2004;80:444-446. doi: 10.1136/pgmj.2003.016873

Sexual health has a profound influence over an individual's physical and psychological wellbeing. There is an increasing need to develop effective strategies to reduce the incidence of sexually transmitted infections (STIs) globally. Many STIs are asymptomatic and yet, highly infectious. The sequelae of such infections may be catastrophic and hence, an integrated multidisciplinary and preventative approach to the control of STIs is clearly necessary. Sexual history taking holds the key to the practice of sexual health medicine. It provides the basis for treatment, prevention, education, and sexual health promotion.

See end of article for authors' affiliations

......................

Correspondence to:

Dr R Jones, Department of

Genito-urinary Medicine,

St Stephen's Centre,

Chelsea and Westminster

Hospital, 369 Fulham

Road, London SW10 9TH,

UK; rachaeljones30@

hotmail.com

Submitted

7 November 2003

Accepted 22 January 2004
S exual health has a profound influence over an individual's physical and psychological wellbeing. As we move further in to the 21 st century, the global ramifications of the HIV pandemic are evident both in social and economic terms and serve to highlight the imperative need for sexual health promotion in order to reduce the burden of sexually transmitted diseases. The UK, like other developed countries, has now begun to recognise the crisis in the provision of sexual services for those requesting screening and treatment. The recognition of the synergistic effect which ulcerative and inflammatory sexually transmitted infections exert on the transmission of HIV have, in the absence of an effective anti-HIV vaccine, led to the increasing need to develop effective strategies to reduce the incidence of sexually transmitted infections (STIs) in both developed and developing countries. These infections have constituted a global health problem for considerably longer than the AIDS epidemic and their prevalence continues to rise.

Health strategies focusing on STIs have previously concentrated on improving treatment, follow up, and contact tracing of those diagnosed with a STI. Treatment decisions are often difficult as exemplified by the therapeutic interventions available for human papilloma virus. Specific strains of this virus have been shown to cause genital warts and treatment modalities are varied-cryotherapy, toxins, and immune modulator agents. But, as with the infections HIV and hepatitis $\mathrm{C}$, there are many conditions that are sexually transmitted which do not cause symptoms yet are infectious, either continuously or intermittently in their natural history. The morbidity and mortality associated with HIV/ AIDS exemplify the severity of the sequelae of such an infection.
In the last century, before the discovery of antibiotics, syphilis was an untreatable condition producing many visible manifestations leading to a similar stigmatisation as seen in HIV today. It is self evident that HIV is just one of many conditions causing silent pathology followed by visible clinical symptoms and signs. However, ectopic pregnancy, hepatic failure, invasive cervical carcinoma, and pelvic sepsis as a sequela of termination of pregnancy cause mortality and morbidity world wide, yet often fail to attract as much media attention or become the focus of public health initiatives (box 1).

\section{CONTROL OF STI}

In light of the above, a more integrated multidisciplinary and preventative approach to the control of STIs is clearly indicated. This has been embodied within the concept "sexual health". The government has produced an "HIV and sexual health strategy", ${ }^{1}$ which advocates effective treatment of symptomatic conditions, and improvement in knowledge and awareness of STIs among the public and health professionals. It hopes to reduce the transmission and prevalence of HIV and STIs, even when undiagnosed, lower unintended pregnancy rates, improve health and social care for those living with HIV, and reduce the stigma associated with STIs. It emphasises the need to improve the multidisciplinary and cross specialty collaboration in detection and management of STIs-for example, screening for STIs in women attending for termination of pregnancy, as well as offering family planning advice to women attending with STIs. Improving access to services by tailoring clinic hours, facilities, and approaches to cater for the public's needs will increase the number of asymptomatic individuals who utilise the service for a check up-for example, walk-in clinics for post-school visits have been designed to increase uptake in the teenage population. Check ups should include, with consent, screening for a whole range of local and systemic infections, as well as providing advice, education, and information with the emphasis on health promotion. Where conditions-symptomatic or asymptomatic-are diagnosed, it is vital that appropriate treatment, follow up, and contact tracing are performed.

The five principles for improvements in sexual health care can be summarised as follows:

(1) Improved access and awareness.

(2) Improved diagnostic techniques.

(3) Improved therapies.

(4) Improved follow up and contact tracing.

(5) Availability of the above to a wider section of the population. 
Box 1: Examples of sexually acquired conditions which may be asymptomatic or subclinical during their natural history, but cause significant morbidity and/or mortality

- HIV infection $\rightarrow$ immunosuppression $\rightarrow$ AIDS.

- Hepatitis B infection $\rightarrow$ chronic hepatitis $\rightarrow$ hepatoma.

- Hepatitis C infection $\rightarrow$ cirrhosis $\rightarrow$ hepatocellular cancer.

- Sperm $\rightarrow$ unwanted pregnancy $\rightarrow$ termination $\rightarrow$ sepsis.

- Oncogenic human papilloma virus cervical infection $\rightarrow$ cervical intraepithelial neoplasia $\rightarrow$ cervical cancer.

- Chlamydia trachomatis $\rightarrow$ pelvic inflammatory disease $\rightarrow$ ectopic pregnancy/infertility.

- Treponema pallidum $\rightarrow$ latency $\rightarrow$ tertiary syphilis.

Finally, sexual health involves more than merely educating and informing patients to change their ideas about the prevention and treatment of STIs. It is vital that medical professionals of any specialty should not see sexual matters as being purely in the realm of the genitourinary medicine clinics, dermatovenereologists, gynaecologists, or urologists. This matter has been discussed extensively as detailed in the British Medical Journal editorial by Stedman and Elstin. ${ }^{2}$ It is most likely that a range of approaches with a common goal will be individually tailored to the needs of different local populations and the resources available. Primary care and accident and emergency physicians, in conjunction with those working in the above specialties, need to work in combination, utilising their expertise to improve the sexual health of the nation.

\section{SEXUAL HISTORY TAKING}

The most important aspect of taking a sexual history lies in the concept that the patient needs to be reassured that his/ her privacy and confidentiality are paramount (box 2). It is essential to ensure that the conversation cannot be overheard or interrupted. Only experience and skill in taking a sexual history can sympathetically encourage patients to reveal the intimate details of their private life that are necessary to determine the choice of examination, laboratory investigations, diagnosis, follow up and, in particular, contact tracing. All information sought should be justifiable on one or other of these grounds. In some cases, it is often helpful to be explicit about these reasons, to allay the patient's fear or embarrassment evoked by these questions. The standard proforma for sexual history taking is shown below (box 3).

\section{Box 2: Principles involved in taking a sexual}

history

- Ensure privacy and confidentiality.

- Be professional.

- Be open minded and non-judgmental.

- Recognise non-verbal cues.

- Ask only appropriate questions.

- Explain procedures and treatments thoroughly.

- Use the time to promote risk reduction and sexual health.
Box 3: Sexual history taking: an example of a structured approach

(1) When did you last have sexual intercourse?

(2) With a man or a woman?

(3) Were they a casual or regular partner?

(4) Where were they from?

(5) In which country did you have sex?

(6) What kind of sex did you engage in?

(7) For each type-for example, oral/vaginal/anal-did you use a condom? (For heterosexual sex: was any contraception used? Relate to risk of pregnancy when inquiring about last menstrual period in gynaecology history).

(8) Does your partner have any symptoms?

(9) Have you had any other partners in the last six weeks? If so, return to question 2. Offer questions which may lead to other problem identification.

(10) Did you have pain during or after intercourse?

(11) Have you ever had any previous STIs (may require information or counselling)?

(12) Have you ever had a sexual health check up before (explain and offer)?

(13) Have you ever had an HIV/hepatitis/syphilis test before (explain and offer, pre-test counsel if appropriate)?

(14) Have you been vaccinated against hepatitis $A / B$ or have you ever had hepatitis (assess risk and offer if appropriate)?

It is important to understand why certain questions should be asked during a consultation. Often, it is useful to pre-empt the patient's discomfort by initially informing them that you are about to ask very personal, and often embarrassing, questions. The early questions should be used to determine a person's sexuality, thus exploring the patient's risk of STI acquisition, appropriate risk reduction information, and offering vaccination for hepatitis $\mathrm{A} / \mathrm{B}$ if appropriate. It is important to define when the last occasion of sexual intercourse occurred as STIs have various incubation and "window periods" and hence, symptoms and signs may be delayed.

STIs, such as the gonococcus, have markedly variable prevalence and antibiotic resistance in differing geographical areas; hence it is useful to determine the ethnicity of the patient's partners and the country in which sexual intercourse took place. Specific details regarding the type of intercourse undertaken and detailing condom use facilitates comprehensive risk assessment and sample acquisition during the STI screening process. It is often useful to use this juncture to discuss safer sex/pregnancy risks/family planning.

In asking the patient if they experience symptoms during sexual intercourse, the physician is able to tailor the consultation appropriately and organise further investigations as necessary. It is imperative to obtain knowledge of the patient's previous sexual history with regard to previous STIs and sexual health screening. Each patient attending the clinic should be offered a blood test for syphilis, HIV, and hepatitis $\mathrm{A} / \mathrm{B} / \mathrm{C}$ if appropriate. Pre-test counselling should be given where necessary. 


\section{Why is sexual history taking so important?}

Sexual history taking holds the key to the proper practice of genitourinary medicine for several reasons. It provides the firm base for gaining information regarding the patient's risks and routes of acquiring an infection as well as setting the agenda for issues concerning specific risks to be further explored. It does this in an environment where the patient is reassured concerning the doctor's professionalism and the maintenance of confidentiality and privacy in the clinic situation. It also raises issues for patients, who will often interject with questions of their own regarding STIs and their acquisition. As such, sexual history taking, although only taking a few minutes, can often be the most potent stimulus for the transmission of information, counselling, and support to the patient beside you.

It would be wrong to say that any individual physician will ever be completely skilled in sexual history taking. Within the clinical situation, there will always be an element of risk in raising subjects which the patient may find difficult or painful to discuss. The well prepared and trained physician will be sympathetic and understand the sensitivity with which questions must be asked. Occasionally, it may be necessary to terminate the history taking until a second visit, or even later, during follow up when the patient is more prepared to provide details of their history. In some cases, patients may never find this possible. Even in such cases, it is paramount to gain as much information, screen for as many infections, contact trace, and provide as much follow up as possible. Only by these methods can the best attempts be made at improving the sexual health of our patients, their partners and their partners' past, present, and future sexual partners.

In different settings, such as primary care, sexual history taking may be much more difficult to achieve. The varied skills of doctors, time constraints, and perceptions of confidentiality may all influence both general practitioners and their patients. This has been clearly analysed in an excellent study by Curtis et al. ${ }^{3}$ Until improved awareness of sexual problems, STIs, and the importance of people's sex lives impacts on more elements of practice in hospitals, primary care and other settings, attempts to improve the nation's sexual health will be fragmented.

\section{EDUCATION IN SCHOOLS}

One of the most important issues influencing the sexual health of the nation will be sex and HIV education in schools. Despite occasional episodes of media outrage complaining of inappropriate sex education "encouraging" teenagers to experiment in sexual behaviour, great improvements have been made for the provision of sex and HIV/AIDS education in schools and these have been shown not to hasten the onset of sexual experience. ${ }^{4}$ Clearly, comprehensive sexual health education can only be of use if it starts before the onset of sexual activity. A study in 2001 found young people lack knowledge of STIs, finding them more aware of the risks of unwanted pregnancy. ${ }^{5}$ The Major National Survey of Sexual Attitudes and Lifestyles revealed that $50 \%$ of young people have had their first sexual experience by the age of $16 .{ }^{6}$ It also revealed that by the time they reach their late teens or early twenties, $40 \%$ of men and $80 \%$ of women who had sex in their early teens wish they had waited longer. Sexual education school lessons were found to be the main source of information regarding sexual matters. It is vital that health care professionals recognise this most important part of the drive towards improved sexual health.

\section{CONCLUSION}

The concept of sexual health emphasises the need to promote health by education and information. It recognises the fact that many STIs including HIV are asymptomatic for a considerable part of their natural history and encourages a further step in the public and medical acceptance that sexual activity is and should be a healthy and fulfilling behaviour. Furthermore, with clear evidence that the treatment of STIs in a developing country can significantly reduce the acquisition of new HIV infection, ${ }^{7}$ it is vital that financial resources designed to decrease the spread of HIV infection should be targeted at improving sexual health and reducing the number of STIs in general.

\section{Authors' affiliations}

R Jones, S Barton, Department of Genito-urinary Medicine, St Stephen's Centre, Chelsea and Westminster Hospital, London, UK

\section{REFERENCES}

1 Department of Health. National strategy for sexual health and HIV. London: Department of Health, 2001.

2 StedmanY, Elstin M. Rethinking sexual health clinics. BMJ 1995;310:342-3.

3 Curtis H, Hoolaghan T, Jewitt C, eds. Sexual health promotion in general practice. Oxford: Radcliffe Medical Press, 1995.

4 Wellings K Wadsworth J, Johnson AM, et al. Provision of sex education and early sexual experience: the relationship examined. BMJ 1995;311:417-20.

5 Garside R, Ayres R, Owen M, et al. "They never tell you about the consequences": young people's awareness of sexually transmitted infections. Int J STD AIDS 2001;12:582-8.

6 Johnson AM, Mercer CH, Erens B, et al. Sexual behaviour in Britain: partnerships, practices, and HIV risk behaviours. Lancet 2001;358:1835-42. 7 Grosskurth H, Mosha F, Todd J, et al. Impact of improved treatment of sexually transmitted diseases of HIV infection in rural Tanzania: randomised controlled trial. Lancet 1995;ii:530-6. 